\title{
Intrinsic abnormal electromagnetic medium based on polar lattice vibration
}

\author{
WANG Rui ${ }^{1}$, ZHOU Ji $^{1 *} \&$ QIU XiangGang ${ }^{2}$ \\ ${ }^{1}$ State Key Laboratory of New Ceramics and Fine Processing, Department of Materials Science and Engineering, Tsinghua University, Beijing \\ 100084, China; \\ ${ }^{2}$ National Laboratory for Superconductivity, Institute of Physics and National Laboratory for Condensed Matter Physics, Chinese Academy of \\ Sciences, Beijing 100080, China
}

Received November 1, 2010; accepted January 27, 2011

\begin{abstract}
A new class of media with abnormal electromagnetic parameters has been attracting increasing attention because of its exotic properties and potential application. Currently, typical metamaterials are mainly composed of artificially designed metallic periodic structures. However, due to the limitations of available fabrication technologies, physical size and material effects, it is difficult to realize these abnormal properties by these artificial structures in the high-frequency regime. Therefore, it is important to find materials with intrinsic abnormal electromagnetic responses. In this field, a new mechanism based on the interaction between polar lattice vibrations and electromagnetic waves has been proposed. In this paper, we review progress in this field.
\end{abstract}

abnormal electromagnetic medium, polar lattice vibration, intrinsic property, infrared, indefinite medium

Citation: Wang R, Zhou J, Qiu X G. Intrinsic abnormal electromagnetic medium based on polar lattice vibration. Chinese Sci Bull, 2011, 56: 1318-1324, doi: $10.1007 / \mathrm{s} 11434-011-4428-9$

Abnormal electromagnetic (EM) medium refer to a kind of material system with abnormal permittivity and permeability (negative or less than unity), which is typically represented by left handed material (LHM) with negative permittivity and permeability at the same time. Abnormal EM medium is normally realized via metamaterials based on metallic periodic structures [1-12]. The abnormal properties in the metamaterial usually originate from the artificial structure, and not from the materials themselves. These materials attracted much attention not only because of their scientific significance, but also their potential for enlargement of the performance scale, and design of new functional materials. Therefore, the initial investigations of LHMs and metamaterials have been honored as breakthroughs in 2003 and 2006, respectively, by Science magazine [13,14]. Also, they were elected to be one of the top ten breakthroughs in material science by the journal, Materials Today [15].

*Corresponding author (email: zhouji@ mail.tsinghua.edu.cn)
Conventional metamaterials are based on highly engineered artificial structure units, normally with electric or magnetic resonances, such as wires and split ring resonators (SRRs) [2,4-9]. In the infrared and optical regions, metamaterials are showing more and more shortcomings for the current fabrication technologies, characteristic physical size and material effects of metals in mesoscopic scale. However, intrinsic EM responses in nature existed materials provide another choice. For example, ferromagnetic resonances in ferrites can provide tunable negative permeability at microwave frequency [16-18], and the electric resonance in ferroelectric can be used to achieve a negative permittivity $[19,20]$. However, because of their high frequency cutoffs, these EM resonances cannot be extended to the infrared or optical regions, restricting the development of intrinsic abnormal media. Therefore, it is a challenging problem to realize intrinsic abnormal EM properties in the high frequency regime. Recently, abnormal EM media based on polar lattice vibrations in the infrared region have attracted 
increasing attention, and significant discoveries have been made. In this paper, we focus on the latest researches and give a review of the field.

\section{Polar lattice vibration and abnormal permittivity}

In the 1950s, Born et al. [21] considered the coupling between a polar lattice vibration mode and EM wave, and quantitatively described the dielectric dispersion relation. For a single oscillator, the dielectric dispersion relation of polaritonic materials can be expressed as

$$
\varepsilon=\varepsilon_{\infty}+\frac{\left(\varepsilon_{0}-\varepsilon_{\infty}\right) \omega_{\mathrm{T}}^{2}}{\omega_{\mathrm{T}}^{2}-\omega^{2}+i \gamma \omega}
$$

where $\varepsilon_{\infty}$ is the high-frequency dielectric constant; $\varepsilon_{0}$ is the static permittivity; $\omega_{\mathrm{T}}$ is the transverse optical phonon frequency; and $\gamma$ is the damping coefficient.

Figure 1 shows the theoretical dielectric dispersion curve of a single vibration mode. The curves can be divided into two parts, one is the region of high permittivity and the other is the region of abnormal permittivity. When the frequency is a little lower than $\omega_{\mathrm{T}}$, the real part of permittivity is high. However, when the frequency is a little greater than $\omega_{\mathrm{T}}$ and satisfies that $\omega_{\mathrm{T}}<\omega<\omega_{\mathrm{L}}$, the real part of the permittivity is less than zero. $\omega_{\mathrm{L}}$ is the longitude optical phonon frequency, which usually corresponds to zero in the dispersion curve. When the frequency is greater than $\omega_{\mathrm{L}}$, the permittivity is less than one. Actually, the region $(\varepsilon<1)$ is the abnormal permittivity region we focus on. In addition, $\omega_{\mathrm{T}}$ and $\omega_{\mathrm{L}}$ satisfy the Lyddane-Sachs-Teller (LST) relation $\omega_{\mathrm{L}} / \omega_{\mathrm{T}}=\sqrt{\varepsilon_{0} / \varepsilon_{\infty}}$, which implies that a much wider abnormal permittivity region can be gained using materials with high $\varepsilon_{0}$ and low $\varepsilon_{\infty}$. If there are $1 / v_{a}$ ion pairs in unit volume, the value of $\omega_{\mathrm{T}}$ depends on the composition of the crystal, and is given by:

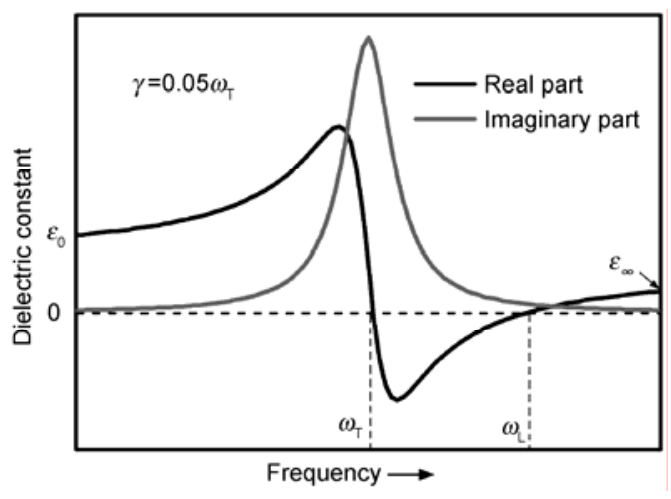

Figure 1 Dielectric dispersion curve for a single vibration mode.

$$
\omega_{\mathrm{T}}=\frac{2}{3}\left(\frac{\pi}{\bar{M} v_{a}\left(\varepsilon_{0}-\varepsilon_{\infty}\right)}\right)^{1 / 2} \operatorname{Ze}\left(\varepsilon_{\infty}+2\right),
$$

where $\bar{M}$ is the reduced mass in a primitive cell and $\mathrm{Ze}$ is the effective charge of the ions. $\omega_{\mathrm{T}}$ is related to the properties of the anion and cation, including mass and electronegativity. In another word, by changing the composition of the material, the value of $\omega_{\mathrm{T}}$ can be controlled. Furthermore, this can yield a tunable abnormal permittivity. Similarly, it can also be extended into other crystals with complex structures. In this case, the dielectric dispersion is modeled by the sum of independent damped harmonic oscillators $[21,22]$ :

$$
\varepsilon=\varepsilon_{\infty}+\sum_{i} \frac{\Delta \varepsilon_{i} \omega_{i \mathrm{~T}}^{2}}{\omega_{i \mathrm{~T}}{ }^{2}-\omega^{2}+i \gamma_{i} \omega},
$$

where the oscillator parameters $\Delta \varepsilon_{i}, \omega_{i \mathrm{~T}}$, and $\gamma_{i}$ are the frequency, oscillator strength, and damping coefficient of the $i$ th transverse vibration mode, respectively.

The abnormal EM medium can be realized both in regions of high permittivity and abnormal permittivity via the interaction between polar lattice vibrations and EM waves. We will give detailed descriptions of these two mechanisms.

\section{Infrared abnormal EM media with tunable permittivity}

The abnormal permittivity can be realized by the polar lattice vibration, and regions with abnormal properties have relations with the composition of the material as descript in Section 1 . Therefore, a tunable abnormal permittivity can be achieved by changing the composition of the crystal using the methods of solid solution or doping. A series of $\mathrm{Ca}_{1-x} \mathrm{Sr}_{x} \mathrm{~F}_{2}$ solid solutions were chosen. Their room temperature reflectance spectra were measured at near-normal incidence in the infrared region. The dielectric properties were measured using the relation between the permittivity and reflectivity, $R=|(\sqrt{\varepsilon}-1) /(\sqrt{\varepsilon}+1)|^{2}$, for non-magnetic materials. The dielectric dispersion curves retrieved from the analysis of the reflectivity data are shown in Figure 2. Because of the interaction between the polar lattice vibrations and the EM waves, the real parts of the permittivity show a resonance mode and the position of the dielectric resonance shifts towards lower frequency as the composition of the material changes from $\mathrm{CaF}_{2}$ to $\mathrm{SrF}_{2}$. For the pure crystals, the dielectric resonance is sharp. The resonance strength of $\mathrm{SrF}_{2}$ is a little stronger than that of $\mathrm{CaF}_{2}$, because the electronegativity of $\mathrm{Sr}$ ions is larger than that of $\mathrm{Ca}$ ions. However, for the solid solution, the partial substitution of $\mathrm{Ca}$ ions with $\mathrm{Sr}$ ions will cause distortions in the $\mathrm{CaF}_{2}$ lattice, which may enhance the damping factor during 

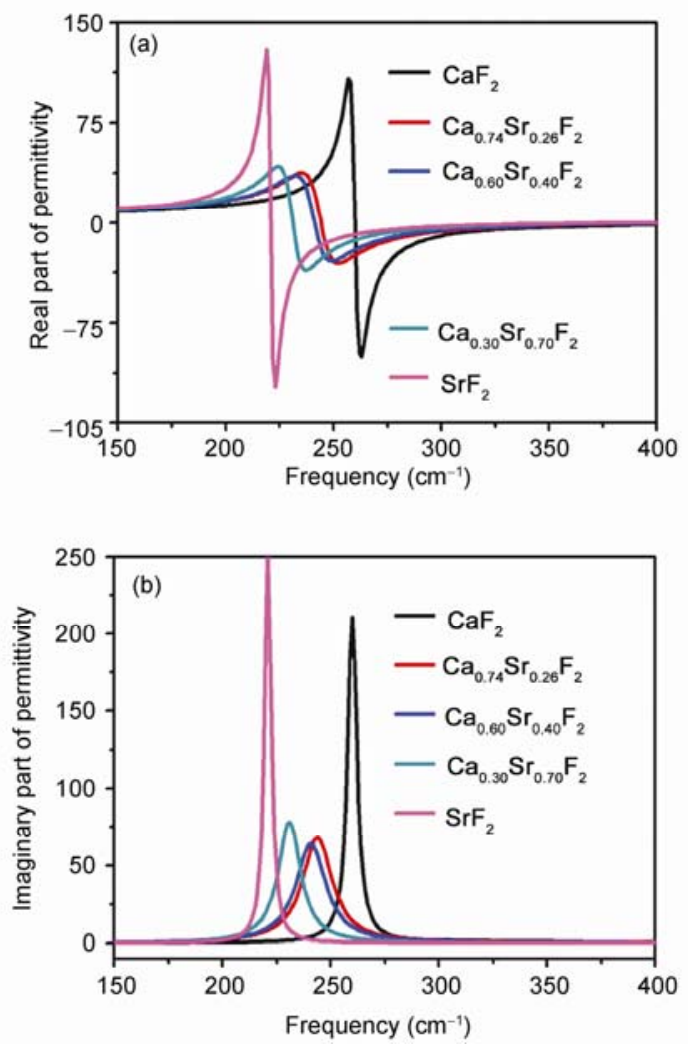

Figure 2 (a) Real and (b) imaginary parts of the dielectric dispersion curves of $\mathrm{Ca}_{1-x} \mathrm{Sr}_{x} \mathrm{~F}_{2}$ with different $x$ values. Modified from [23].

the vibration and result in a larger dissipation. As a result, the resonance strength of the solid solutions decreases significantly. When the composition is close to $1: 1$, the resonance strength reaches its minimum.

Figure 3 presents the permittivity of $\mathrm{Ca}_{1-x} \mathrm{Sr}_{x} \mathrm{~F}_{2}$ as a function of composition at three typical frequencies, 330, 480, and $620 \mathrm{~cm}^{-1}$. It shows that as the molar fraction $x$, varies from 0 to 1 , the variation range of the permittivity can cover part of the negative region, the whole of the ultra-low (zero to one) region, and reaches permittivity of one. Additionally, it can be seen from Figure 3 that when the frequency is close to the transverse optical frequency, the dielectric loss is enhanced. The large dielectric loss will cause a strong absorption of EM waves in the material, which is unfavorable for the application. Therefore, the working frequency should be far from the resonance frequency when using the polar lattice vibration to realize abnormal permittivity.

\section{Abnormal EM media composed of high- permittivity particles based on Mie theory}

In the paper published by Born et al. [24] in 1908, they derived a rigorous solution for plane EM wave diffraction by spherical particles. Combining Mie theory with effective medium theory, Lewin [25] investigated the EM responses
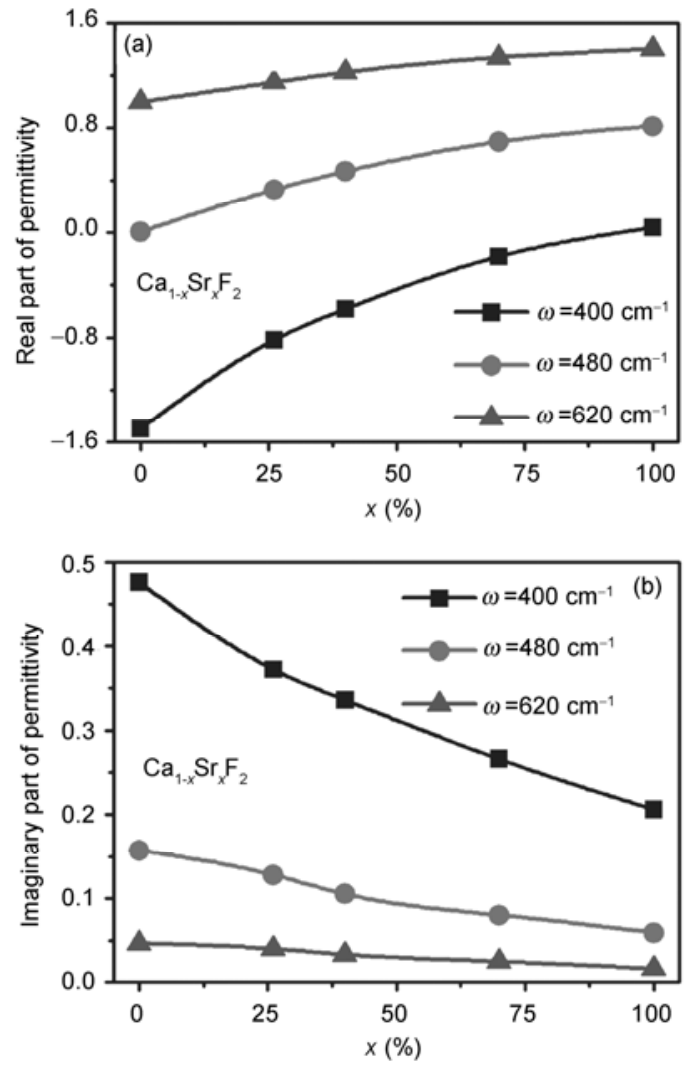

Figure 3 (a) Real and (b) imaginary parts of the permittivity of $\mathrm{Ca}_{1-x} \mathrm{Sr}_{x} \mathrm{~F}_{2}$ at 400, 480, and $620 \mathrm{~cm}^{-1}$. Modified from [23].

of the composite with dielectric or magnetic spherical particles dispersion in another background matrix, and derived the effective permittivity and permeability:

$$
\begin{gathered}
\varepsilon_{\text {eff }}=\varepsilon_{\mathrm{h}}\left[1+\frac{3 v}{\frac{F(\theta)+2 K_{\mathrm{e}}}{F(\theta)-K_{\mathrm{e}}}-v}\right], \\
\mu_{\mathrm{eff}}=\mu_{\mathrm{h}}\left[1+\frac{3 v}{\frac{3(\theta)+2 K_{\mathrm{m}}}{F(\theta)-K_{\mathrm{m}}}}\right],
\end{gathered}
$$

where $K_{\mathrm{e}}=\varepsilon_{\mathrm{h}} / \varepsilon_{\mathrm{p}}$ and $K_{\mathrm{m}}=\mu_{\mathrm{h}} / \mu_{\mathrm{p}} . \varepsilon_{\mathrm{p}}$ and $\mu_{\mathrm{p}}$ are the permittivity and permeability of particles, respectively. $\varepsilon_{\mathrm{h}}$ and $\mu_{\mathrm{h}}$ are the permittivity and permeability of the matrix, respectively. $v$ is the volume fraction of the spherical particles; $F(\theta)=$ $2(\sin \theta-\cos \theta) /\left[\left(\theta^{2}-1\right) \sin \theta+\theta \cos \theta\right] ; \quad \theta=k_{0} r \sqrt{\varepsilon_{\mathrm{p}} \mu_{\mathrm{p}}} ; r$ is the radius of the particles; and $k_{0}$ is the free-space wavenumber. It can be seen that, $F(\theta)$ becomes negative infinite when its denominator nears zero, which results in either a negative permittivity or permeability. Figure 4 shows the effective EM parameters for the composite. 


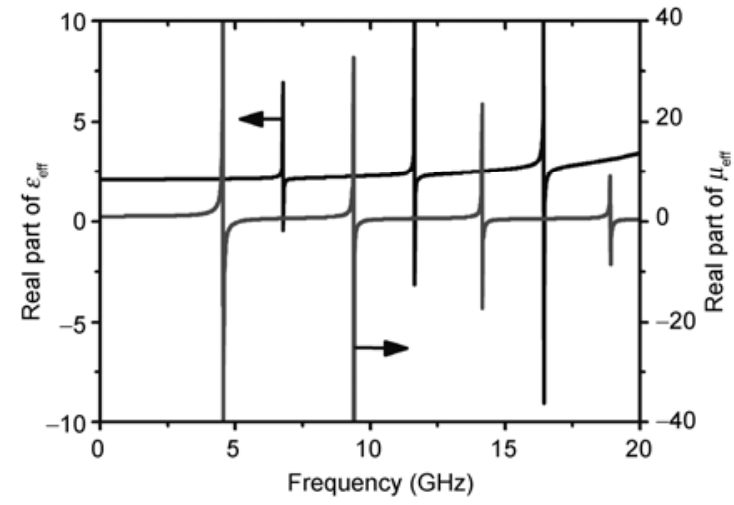

Figure 4 Effective EM parameters of the composite. $\varepsilon_{\mathrm{p}}=100 ; \mu_{\mathrm{p}}=\varepsilon_{\mathrm{h}}=\mu_{\mathrm{h}}=1$; $v=0.064$.

For Mie resonances, particles with a large permittivity or permeability are required. However, the permittivity and permeability of most materials will decrease as the frequency increases and cannot maintain a high level. The large permittivity present during the lattice vibrations provides a promising way to realize Mie resonances at a high frequency. Thus, it allows for an abnormal EM medium in the infrared region. Wheeler et al. [26] proposed a scheme to obtain negative effective permeability using a collection of polaritonic spheres. Based on the polar lattice vibration of $\mathrm{LiTaO}_{3}$ crystal, which result in a high permittivity at infrared frequency, the effective parameters were calculated and the results are shown in Figure 5.

Later, Wheeler et al. [27] also proposed a core-cell structure, with a $\mathrm{LiTaO}_{3}$ sphere core and a coating on its surface whose dispersion relation follows the Drude model. Using this structure they achieved an isotropic LHM in far infrared region. Schuller et al. [28] experimentally observed the abnormal EM properties of SiC crystal whiskers based on Mie resonance. They also proposed that it could be possible to obtain a LHM by combining the $\mathrm{TE}_{011}$ and $\mathrm{TM}_{011}$ modes using $\mathrm{SiC}$ particles of two sizes.

In addition, by selecting suitable polaritonic crystals, this kind of abnormal EM medium can be achieved in other infrared regions.

\section{All-dielectric LHM at infrared frequency}

LHM is a kind of metamaterials, whose permittivity and permeability are both less than zero. Similarly, theoretical and experimental researches primarily focus on the artificial periodic metallic structures, which are typically represented by the composition of SRRs and metallic wires [4-9,2933]. In terms of intrinsic properties of materials, a common method is to use the polariton as an electric inclusion to provide negative permittivity. This can be combined with dielectrics which possess negative permeability. In this case, an all-dielectric LHM can be achieved without metallic structures.
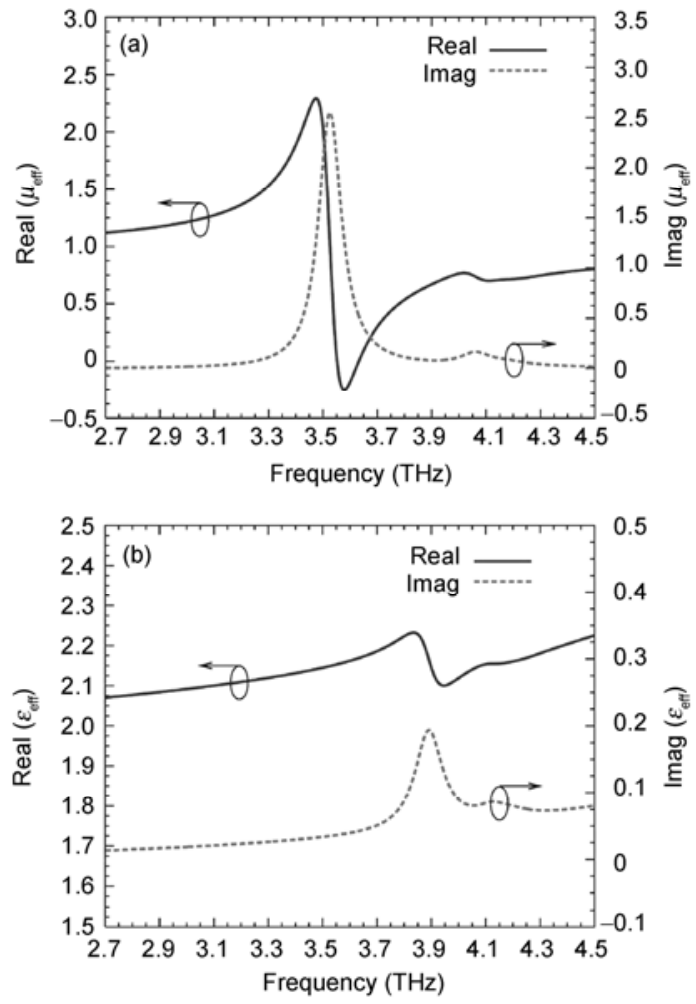

Figure 5 Effective (a) permeability and (b) permittivity of $\mathrm{LiTaO}_{3}$ spheres. Reprinted figure with permission from [26].

Based on the cation- $\mathrm{TiO}_{3}$ lattice vibration mode, which is located at about $110 \mathrm{~cm}^{-1}$, to provide a negative permittivity, our group selected $\mathrm{CaTiO}_{3}$ (CTO) rods as electric inclusion. By combining the CTO rods with $\mathrm{LiTaO}_{3}$ (LTO) spheres based on Mie magnetic resonance, we realized an all-dielectric LHM at $3.5 \mathrm{THz}$ [34]. The EM transmission behaviors were simulated using the CST microwave studio based on the finite-difference time-domain (FDTD) method. The effective EM parameters, which were retrieved according to effective medium theory, are shown in Figure 6. There is a passband, which is marked with a blue arrow, near the overlap of the forbidden bands of the LTO spheres and the CTO rods. The passband is a left-handed band, in which the effective permittivity, permeability, and the refractive index of the composite are all less than zero. The effective region with negative index is $3.53-3.64 \mathrm{THz}$. The minimum value, which is about -1 , is achieved at $3.54 \mathrm{THz}$.

The electric and magnetic inclusions are not isolated and they interact with each other. Using EM simulation, the coupling effects between them were investigated [34]. The results show that when lattice vibrations occur in the CTO rod, a displacement current forms inside the CTO rod, which results in an annular induced magnetic field. There is an interaction between the induced magnetic field and the magnetic resonance field in the LTO sphere, which destroys the magnetic resonance and results in a composite without lefthanded (LH) properties. Therefore, the distance between the 

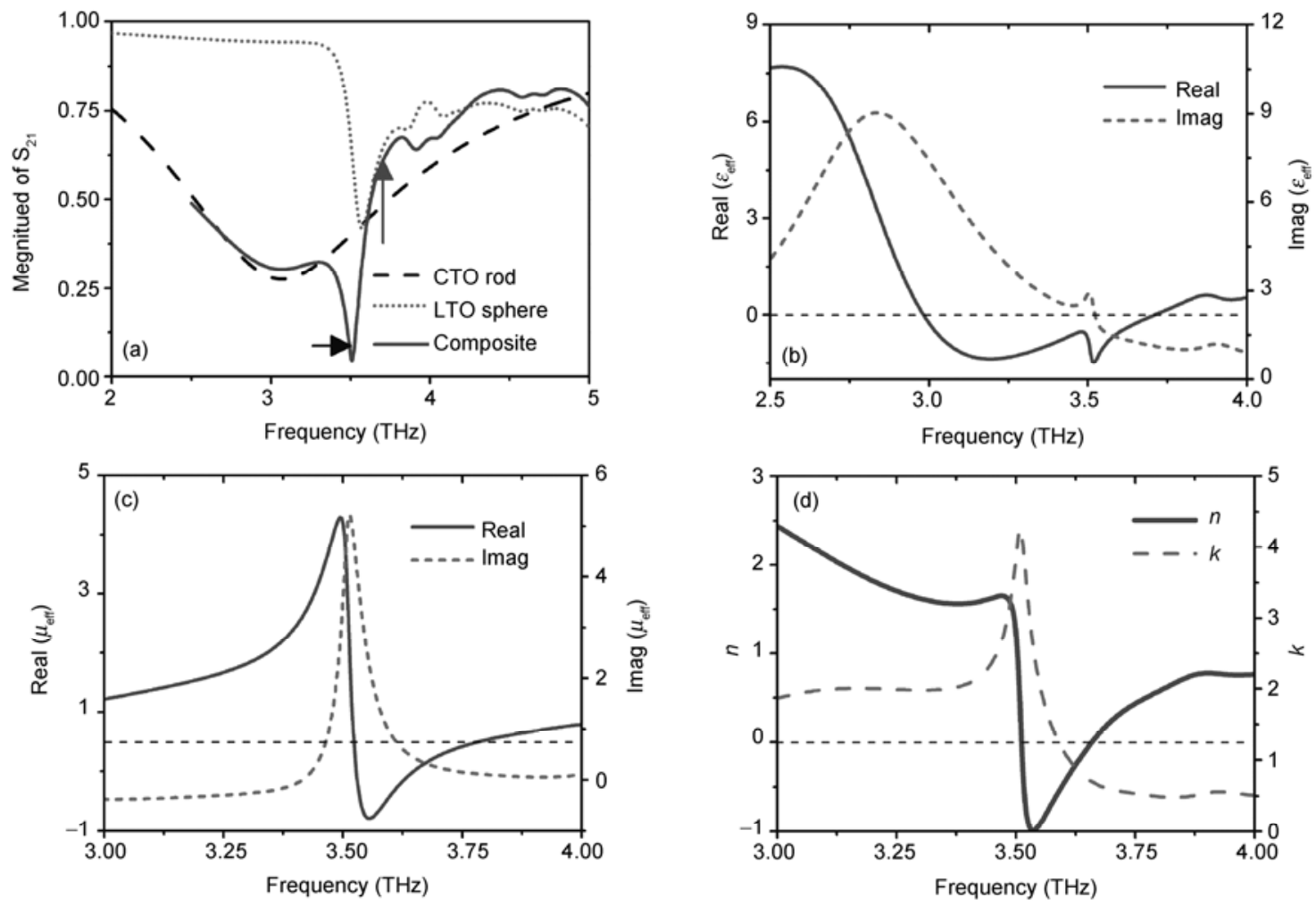

Figure 6 Effective EM parameters of the CTO/LTO composite. Reprinted by permission of [34].

electric and magnetic inclusions, the intrinsic dielectric resonance frequency of the electric inclusions, and the propagation direction of the EM wave all affect the LH properties of the LTO/LTO composite [34].

\section{$5 \quad$ Indefinite medium based on anisotropic lattice vibrations}

Negative refraction is a fantastic phenomenon that when an EM wave is incident from a medium to another and the incident light and refractive light are located on the same side of normal direction. For a long time, the negative refraction has been considered as a unique characteristic of LHM [1,3-7], until Lindell et al. [35] investigated the optical properties of anisotropic media and proposed a kind of indefinite medium, a non-LH negative refraction was realized. Indefinite medium is a kind of abnormal EM medium. It is also a kind of uniaxial and anisotropic medium, in which only one of the four components of $\varepsilon$ and $\mu$ is negative, while the others are positive [35-37]. If only consider the e light with a wave vector $\boldsymbol{k}$ propagating in the $x z$ plane, and the non-magnetic medium satisfies $\varepsilon_{\mathrm{x}}>0$ and $\varepsilon_{\mathrm{Z}}<0$, then the dispersion relation in the medium is as follows:

$$
\left(\frac{\omega}{c}\right)^{2}=\frac{k_{\mathrm{z}}^{2}}{\left|\varepsilon_{\mathrm{x}}\right|}-\frac{k_{\mathrm{x}}^{2}}{\left|\varepsilon_{\mathrm{z}}\right|},
$$

where the subscript $x$ or $z$ refers to the components of the vector in the corresponding direction. In this case, the equifrequency surface (EFS) in the crystal has a hyperbolic dispersion relation. The refracted directions of the wave vector and Poynting vector do not lie in the same side of the normal direction, thus a negative refraction of light. This theory has already been confirmed by experiments and simulations [36]. The angles of refraction for the wave vector and Poynting vector are given by $\theta_{\mathrm{k}}=\tan ^{-1}\left(k_{\mathrm{x}} / k_{\mathrm{z}}\right)$ and $\theta_{\mathrm{s}}=\tan ^{-1}$ $\left(S_{\mathrm{x}} / S_{\mathrm{z}}\right)=\tan ^{-1}\left(k_{\mathrm{x}} \varepsilon_{\mathrm{x}} / k_{\mathrm{z}} \varepsilon_{\mathrm{z}}\right)$, respectively. At present, the indefinite medium is still achieved by artificial structures, such as metal/dielectric multilayer [37] and metal nanowire arrays [36]. Although many existing materials, such as a $\mathrm{YVO}_{4}$ twin structure [38], can also show negative refraction, this negative refraction can only be achieved over a small region of incident angles (about $0-12^{\circ}$ ). Thus, it is not an all-angle negative refraction. Therefore, we proposed an indefinite medium based on the strong anisotropy of polar lattice vibrations in uniaxial crystals. This will provide a promising method for the realization of all-angle LH properties using intrinsic properties of materials [39].

In a uniaxial crystal, because the parameters of the lattice are different in the two directions, parallel and perpendicular to optical axis, the lattice vibration frequencies for the same vibration mode will be different. This will result in an indefinite permittivity with a strong anisotropy, which is shown in Figure 7. 


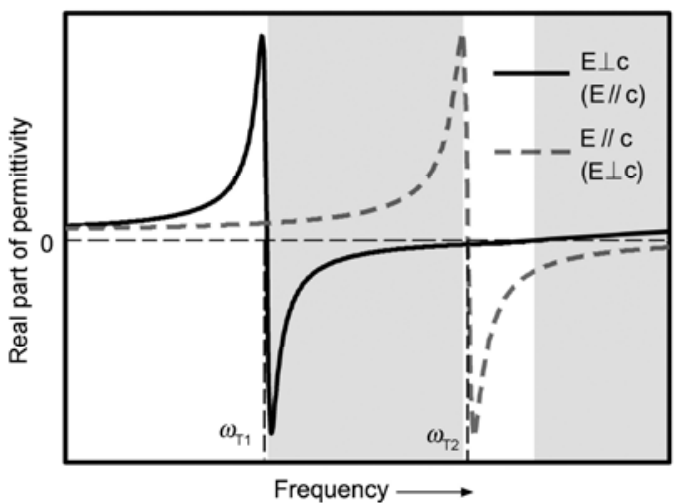

Figure 7 Dielectric dispersion in a uniaxial crystal with a simple structure.

We used $\alpha-\mathrm{Al}_{2} \mathrm{O}_{3}$ single crystal to serve as an example and measured its reflectivities with different polarizations [39]. The retrieved permittivities are shown in Figure 8. Four indefinite permittivity regions are present, and they are indicated by the shaded regions in the figure. In these regions, $\alpha-\mathrm{Al}_{2} \mathrm{O}_{3}$ can be considered as an indefinite medium and is in the family of abnormal EM media.

The magnetic field component in the $y$-direction demonstrates a negative refraction when the $500 \mathrm{~cm}^{-1} \mathrm{EM}$ wave is refracted at the interface between the $\alpha-\mathrm{Al}_{2} \mathrm{O}_{3}$ and vacuum, which is shown in Figure 9. It can be seen that after the refraction of the EM wave at the surface, the wave vector $\boldsymbol{k}$, which simply considered as the propagation direction of the peaks and crests, is refracted positively; however, the Poynting vector, $S$, is refracted negatively. Additionally, the large losses of the permittivity cause a strong absorption. Therefore, the refractive wave can only propagate for several wavelengths in the crystal. This is why the negative refraction is hard to detect.

Figure 10 shows the calculated results of the refracted $\theta_{\mathrm{k}}$ and $\theta_{\mathrm{S}}$ at different incident angles at $500 \mathrm{~cm}^{-1}$. It can be seen that an all-angle negative refraction is achieved using

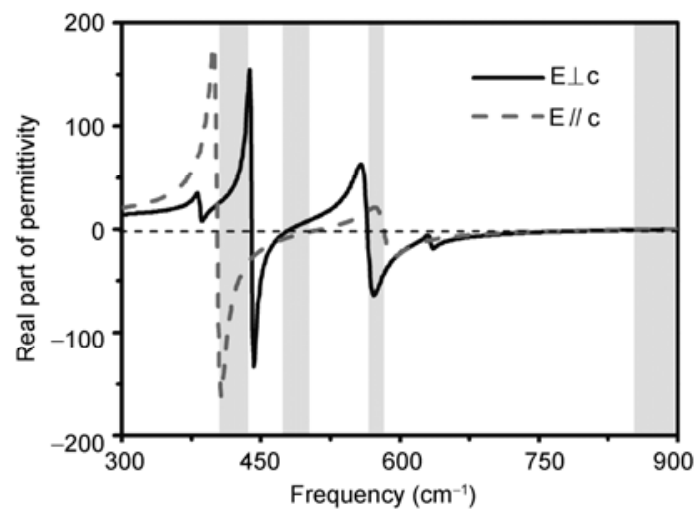

Figure 8 Dielectric dispersion curves of $\alpha-\mathrm{Al}_{2} \mathrm{O}_{3}$ single crystal. Reprinted with permission from [39], Copyright [2010], American Institute of Physics.

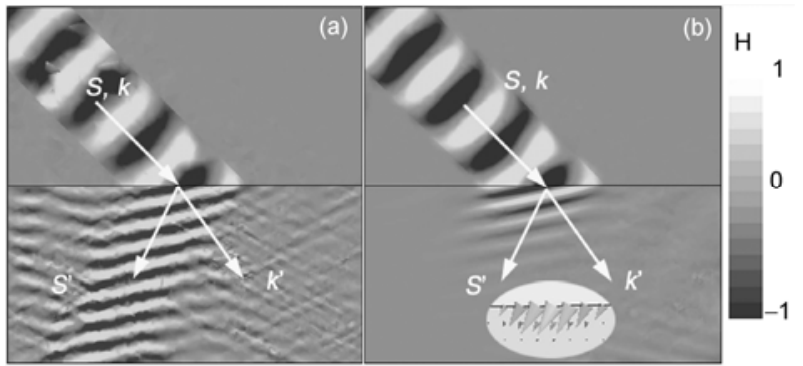

Figure 9 Simulation results for $500 \mathrm{~cm}^{-1} \mathrm{TM}$ light at incident angles of $45^{\circ}$ (a) without and (b) with considering the loss of the permittivity. The $c$ axis is the optical axis and is perpendicular to the interface. The source is a TM Gaussian beam with a width of $20 \mu \mathrm{m} . \varepsilon_{\perp}=7.56+1.90 \mathrm{i}$ and $\varepsilon_{/ /}=$ $-3.14+1.30 \mathrm{i}$.

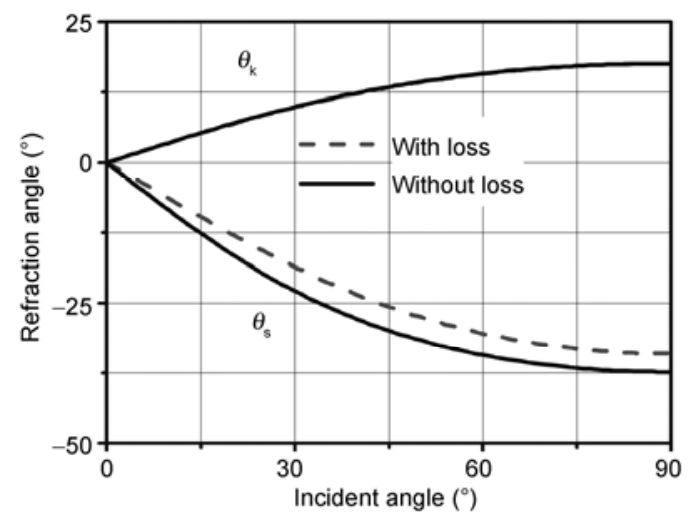

Figure 10 Calculated results of $\theta_{\mathrm{k}}$ and $\theta_{\mathrm{S}}$ at $500 \mathrm{~cm}^{-1}$.

the intrinsic indefinite medium. When the dielectric loss increases in the material, $\theta_{\mathrm{S}}$ and the absolute value of the refractive index will both decrease.

Although we used $\alpha-\mathrm{Al}_{2} \mathrm{O}_{3}$ single crystal as an example, and its region with indefinite permittivity only covers part of the range $400-900 \mathrm{~cm}^{-1}$. However, by selecting other uniaxial crystals with different lattice vibration frequencies, such as $\mathrm{SiC}$ [40], this kind of indefinite medium can be achieved in other infrared regions. Thus, negative refraction can be achieved on a much wider scale.

\section{Summary}

The theory of coupling effects between polar lattice vibrations and EM waves has already been formed over a period of more than half a century. However, the application of this interaction mechanism in functional materials is rarely reported. It is actually a promising method for designing optical abnormal media based on intrinsic properties. The variety of crystal structures in nature provides abundant resources for abnormal dielectrics. By selecting suitable compositions and structures, it is possible to extend the abnormal EM properties over a wide frequency region. 
This work was supported by the National Natural Science Foundation of China (90922025, 51032003, 50921061 and 10774087).

1 Veselago V G. The electrodynamics of substance simultaneously negative values of $\varepsilon$ and $\mu$. Sov Phys Usp, 1968, 10: 509-514

2 Pendry J B, Holden A J, Stewart W J, et al. Extremely low frequency plasmons in metallic mesostructures. Phys Rev Lett, 1996, 76: 47734776

3 Pendry J B. Negative refraction makes a perfect lens. Phys Rev Lett, 2000, 85: 3966-3969

4 Shelby R A, Smith D R, Schultz S. Experimental verification of a negative index of refraction. Science, 2001, 292: 77-79

5 Kolinko P, Smith D R. Numerical study of electromagnetic waves interacting with negative index materials. Opt Express, 2003, 11: 640-648

6 Fang N, Zhang X. Imaging properties of a metamaterial superlens. Appl Phys Lett, 2003, 82: 161-163

7 Shalaev V M, Cai W, Chettiar U K, et al. Negative index of refraction in optical metamaterials. Opt Lett, 2005, 30: 3356-3358

8 Smith D R, Gollub J, Mock J J, et al. Calculation and measurement of bianisotropy in a split ring resonator metamaterial. J Appl Phys, 2006, 100: 024507

9 Yuan H K, Chettiar U K, Cai W, et al. A negative permeability material at red light. Opt Express, 2007, 15: 1076-1083

10 Zhao Q, Lei K, Zhao H, et al. Experimental demonstration of isotropic negative permeability in a three-dimensional dielectric composite. Phys Rev Lett, 2008, 101: 027402

11 Banzer P, Peschel U, Quabis S, et al. On the experimental investigation of the electric and magnetic response of a single nano-structure. Opt Express, 2010, 18: 10905-10923

12 Wang J F, Qu S B, Xu Z, et al. Normal-incidence left-handed metamaterials based on symmetrically connected split-ring resonators. Phys Rev E, 2010, 81: 036601

13 Breakthrough of the year-The runners-up. Science, 2003, 302: 20392045

14 Breakthrough of the year. Science, 2006, 314: 1841

15 The top ten advances in materials science. Mater Today, 2008, 11: 40-45

16 Ueda T, Tsutsumi M. Left-handed transmission characteristics of rectangular waveguides periodically loaded with ferrite. IEEE Trans Magn, 2005, 41: 3532-3537

17 Rachford F J, Armstead D N, Harris V G, et al. Simulations of ferrite-dielectric-wire composite negative index materials. Phys Rev Lett, 2007, 99: 057202

18 Zhao H, Zhou J, Zhao Q, et al. Magnetotunable left-handed material consisting of yttrium iron garnet slab and metallic wires. Appl Phys Lett, 2007, 91: 131107

19 Bai Y, Chen H S, Zhang J J, et al. Left-handed material based on ferroelectric medium. Opt Express, 2007, 15: 8284-8289

20 Zhao H, Kang L, Zhou J, et al. Experimental demonstration of tunable negative phase velocity and negative refraction in a ferromagnetic/ferroelectric composite metamaterial. Appl Phys Lett, 2008, 93 :
201106

21 Born M, Huang K. Translated by Ge W K, Jia W Y. Dynamical Theory of Crystal Lattices (in Chinese). Beijing: Peking University Press, 2006

22 Zhang G Y, Lan G X. Lattice Vibration Spectroscopy (in Chinese). Beijing: Higher Education Press, 1992

23 Wang R, Zhou J, Li B, et al. Intrinsic negative permittivity at far infrared region based on crystal lattice vibration. IEEE Photonics Global Conference, 2010, in press

24 Born M, Wolf E. Translated by Yang J S. Principles of Optics: Electromagnetic Theory of Propagation, Interference and Diffraction of Light (in Chinese). Beijing: Electronic Industry Press, 2006

25 Lewin L. The electrical constants of a material loaded with spherical particles. Proc Inst Electr Eng, 1947, 94: 65-68

26 Wheeler M S, Aitchison J S, Mojahedi M. Three-dimensional array of dielectric spheres with an isotropic negative permeability at infrared frequencies. Phys Rev B, 2005, 72: 193103

27 Wheeler, M S, Aitchison J S, Mojahedi M. Coated nonmagnetic spheres with a negative index of refraction at infrared frequencies. Phys Rev B, 2006, 73: 045105

28 Schuller J A, Zia R, Taubner T, et al. Dielectric metamaterials based on electric and magnetic resonances of silicon carbide particles. Phys Rev Lett, 2007, 99: 107401

29 Smith D R, Padilla W J, Vier D C, et al. Composite medium with simultaneously negative permeability and permittivity. Phys Rev Lett, 2000, 84: 4184-4187

30 Huangfu J, Ran L, Chen H, et al. Experimental confirmation of negative refractive index of a metamaterial composed of $\Omega$-like metallic patterns. Appl Phys Lett, 2004, 84: 1537-1539

31 Smith D R, Pendry J B, Wiltshire M C K. Metamaterials and negative refractive index. Science, 2004, 305: 788-792

32 Economou E N, Koschny T, Soukoulis C M. Strong diamagnetic response in split-ring-resonator metamaterials: Numerical study and two-loop model. Phys Rev B, 2008, 77: 092401

33 Vier D C, Schultz S, Greegor R B, et al. Three-dimensional double-negative metamaterials resonating at $13.5 \mathrm{GHz}$. IET Microw Antennas Propag, 2009, 3: 723-727

34 Wang R, Zhou J, Sun C Q, et al. Left-handed materials based on crystal lattice vibration. Prog Electromagn Res Lett, 2009, 10: 144155

35 Lindell I V, Tretyakov S A, Nikoskinen K I, et al. BW media-media with negative parameters, capable of supporting backward waves. Micro Opt Tech Lett, 2001, 31: 129-133

36 Yao J, Liu Z, Liu Y, et al. Optical negative refraction in bulk metamaterials of nanowires. Science, 2008, 321: 930

37 Yao Y, Huang J P, Liu Y M, et al. Optical negative refraction in ferrofluids with magnetocontrollability. Phys Rev Lett, 2010, 104: 034501

38 Zhang Y, Fluegel B, Mascarenhas A. Total negative refraction in real crystals for ballistic electrons and light. Phys Rev Lett, 2003, 91: 157404

39 Wang R, Sun J B, Zhou J. Indefinite permittivity in uniaxial single crystal at infrared frequency. Appl Phys Lett, 2010, 97: 031912

40 Palik E D. Handbook of Optical Constant of Solid II. New York: Academic Press, 1985

Open Access This article is distributed under the terms of the Creative Commons Attribution License which permits any use, distribution, and reproduction in any medium, provided the original author(s) and source are credited. 